\title{
Improved Hybrid Navigation for Space Transportation
}

\author{
Guilherme F. Trigo and Stephan Theil
}

\begin{abstract}
This paper presents a tightly coupled hybrid navigation system for space transportation applications. The tightly integrated set-up, selected for its robustness and design flexibility, is here updated with GPS Pseudoranges and Time-Differenced GPS Carrier Phases (TDCP) to promote fast-dynamics estimation. The receiver clock errors affecting both GNSS observables are analysed and modelled. Tropospheric delay-rate is found to cause major disturbance to the TDCP during atmospheric ascent. A robust correction scheme for this effect is devised. Performance is evaluated using real GPS measurements.
\end{abstract}

\section{Introduction}

Navigation on-board most launchers and sounding rockets is purely inertial: measurements from strapdown accelerometers and gyroscopes are integrated from an initial condition yielding a high-rate navigation solution. While self-contained and robust, inertial navigation suffers from a fundamental drawback: drift. If uncorrected, the inertial propagation errors will boundlessly grow over time. To compensate for this, inertial sensors are made increasingly accurate, raising cost, and often also volume and mass. Still, inertial drift is unavoidable, posing important performance and operational constraints on both launchers and sounding rockets. Trajectory maintenance and injection greatly depend on navigation accuracy. Not rarely, delivered spacecraft need to perform costly orbital manoeuvres to correct for deficient injection, potentially reducing operation life-time and causing loss of commercial/scientific profit. With drift, a strong limitation is also set on launch mission duration and profile. For instance, return phases in missions of reusable vehicles simply cannot cope with the error inertial propagation yields, requiring other

G. F. Trigo $\cdot$ S. Theil

German Aerospace Center (DLR), Institute of Space Systems, GNC Systems Department, Bremen, Germany, e-mail: guilherme.trigo@dlr.de 
measurement sources [19]. In sounding rockets, with lower budget and operational requirements, when used, inertial sensors are generally of (comparatively) lower grade. Considerable uncertainties in on-board tracking information are thus typical. Moreover, the available propagated inertial attitude is often of poor quality, potentially limiting scientific payloads or forcing these to feature their own solutions.

GNSS receivers have long been used to complement inertial systems. In fact, the two sensors gracefully ease each others' flaws: the GNSS receiver bounds inertial drift, while the high-rate inertial measurements bridge the low-rate GNSS outputs [9]. This combination can be achieved in three main ways: loosely coupled uses receiver navigation solution to correct the inertial solution; tightly coupled avoids filter cascading by using GNSS raw measurements; while ultra-tight (deep for some authors) goes even further by driving the receiver tracking loops with the inertially aided correlators outputs [9]. In this paper we study the application of a tightly coupled GPS/Inertial Measurement Unit (IMU) hybrid system to rocket navigation.

In a launch environment, however, GNSS technology has a set of important vulnerabilities. As non-self-contained, it can suffer external signal disturbance and disruption. Jamming and spoofing, for instance, pose real threat to signal quality and veracity, while ionospheric scintillations can cause temporary signal loss. Additionally, signal tracking is not immune to the high-dynamics, vibration and shocks experienced in-flight. Although complete mitigation of all these risks is not possible, improved receiver design/tuning and integrity monitoring can lessen their effects. Moreover, combination with inertial sensors improves availability and robustness.

Despite the fundamental drawbacks described, which raised considerable opposition to the use GNSS technology in rocket applications, several examples of GNSSbased systems for space transportation have been conceived and tested. In the U.S., the Space Integrated GPS/INS, known as SIGI, a primary navigation system designed by Honeywell under a NASA contract, has been extensively tested including on-board of the Space Shuttle [29]. Also in the U.S., GPS metric tracking systems (GPS-MT), a class of on-board GPS-based tracking and telemetry systems, have been targeted as key technology after decomissioning of C-band radar facilities. Under this initiative, United Launch Aliance has developed a system using COTS components [2], which is routinely flown on Atlas V and Delta IV launchers as main tracking means. Other GPS-MT systems for small launchers and sounding rockets are described by Slivinsky et al. [20] and Williams et al. [28]. In Russia, a hybrid INS/GLONASS/GPS primary navigation system has been tested on-board of the Fregat upper stage. After numerous flights, a 100 -fold improvement was attained in orbital injection accuracy in GTO/GEO missions with respect to purely inertial performance [4]. In Europe, DLR has developed and flight-proved the Orion GPS receiver for rocket applications [15], and more recently the Phoenix-HD receiver [13]. Due to the lack of redundancy in the tracking of the European small launcher VEGA, a COTS GPS receiver is currently flown as part of the ALTS (Autonomous Localization and Telemetry Subsystem). This is planned to be coupled with a COTS inertial sensor in coming flights [6]. In 2014, as part of the OCAM-G experiment, a cooperation between ESA and European national space agencies and industry partners, a set of three GNSS receivers (including the Phoenix) flew on-board of the 
Ariane-V launcher [11]. Results of this experiment have been used by Braun et al. [1] to study an inertially-aided GNSS tracking system. In terms of primary navigation, an extensive study by Airbus DS with ESA funding, the HiNAV, developed a prototype GNSS/INS system for European launch and re-entry vehicles, revealing promising results [18]. Finally, DLR has conceived and successfully flown a tightly coupled GPS/INS system, the Hybrid Navigation System (HNS), on the SHEFEX2 experimental flight. Envisaged as primary navigation, the HNS uses a COTS IMU, a Phoenix receiver and a DLR-developed star sensor [25, 22].

Following the HNS development and SHEFEX2 flight, the Pseudorange-only filter updating was found to yield slow velocity estimation during engine burns [24]. As accuracy is critical during propelled flight phases, both for optimal vehicle steering and for safety monitoring, a GNSS velocity-based measurement should be included. Pseudorange-rate is a common choice, however, Time-Differenced Carrier Phase (TDCP), which is a measure of delta-range, has shown superior performance in highly dynamic applications such as missile [27] and agile UAV [30]. The timedifferencing cancels out the phase ambiguity terms, avoiding the need for ambiguity fixing by differential operation or ambiguity resolution [8]. The application of TDCP observables to the rocket tightly coupled navigation problem is here investigated. Receiver clock error affecting GNSS measurements can be eliminated by channel differencing as done by Steffes [23], however, this reduces measurement availability (as it requires at least two satellites). A reduced order receiver clock model is here described, which takes into account the correlation between clock error in Pseudorange (PR) and TDCP measurements. Furthermore, Tropospheric delay-rate is shown to be a major error source in a rocket ascent scenario. A way of robustly correcting for this effect within the navigation filter is proposed.

This paper begins with a discussion on GNSS/INS architectures in the light of rocket navigation. An explanation on the GPS TDCP observable and the models used for receiver clock error and tropospheric delay precedes a detailed description of the hybrid navigation algorithm. The proposed system performance is then evaluated using real GNSS data.

\section{GNSS/IMU Hybrid Navigation Architectures}

There are several ways to combine (or couple) GNSS measurements with inertial ones, from an IMU or INS. Fig. 1 shows the most common coupling architectures. Excluding the uncoupled configuration, where INS and GNSS each independently provide a navigation solution, the simplest set-up is the loosely coupled (Fig. 1a). This uses the GNSS navigation solution to correct the inertial propagation through a fusion algorithm (e.g. a Kalman filter) [9]. The tightly coupled architecture (Fig. 1b), instead, uses raw GNSS measurements (e.g. Pseudorange, Pseurange-rate, Carrier Phase), allowing the navigation filter to still draw information from sets of fewer than four tracked satellites. This is very important given the tracking vulnerabilities of GNSS under rocket dynamics. A less obvious advantage of the tightly coupled 


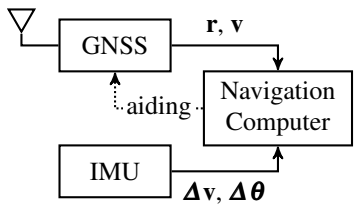

(a) Loose

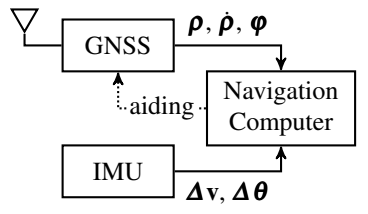

(b) Tight

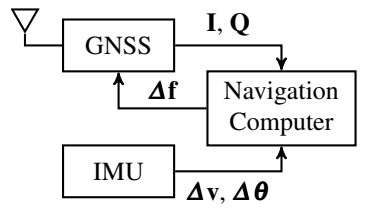

(c) Ultra-tight

Fig. 1 IMU/GNSS hybridization architectures.

scheme is the avoidance of a cascade of two filters: the GNSS receiver fix is obtained through filtering, making it heavily time-correlated with a time-varying statistical profile, which may easily lead to severe mismodelling, degrading robustness and performance [9]. In these two configurations the fused estimates can be provided as aiding to the GNSS receiver to expedite SV (re)acquisition. Finally, in an ultra-tight coupling (Fig. 1c) the receiver accumulated correlator outputs are fused with the inertial measurements, and fed back to drive the receiver tracking loops. This scheme achieves the best performance, however, it requires extensive access to the internal functioning of the receiver or parallel development of both receiver and hybrid system. The design complexity of such a system is thus far greater than that of the less coupled options. The tightly coupled set-up is chosen for this study for allowing the use of COTS components while still yielding strong levels of performance and robustness.

A further design option has to do with the open-/closed-loop nature of the estimated navigation corrections [9]. In the open-loop configuration the fusion algorithm estimates corrections to be applied to the inertial propagator output (position, velocity and attitude). These corrections may grow indefinitely as the inertial solution drifts. Instead, the inertial propagation may be regularly reset using the fused estimates in a closed-loop set-up. This makes sure that the filter remains close to the origin, reducing linearization errors and numerical issues due to unbounded state growth. Moreover, it allows for the inertial sensor online calibration, offering higher levels of performance and robustness. This option was adopted in this study.

Within the closed-loop set up, the level of modularity between fusion filter and inertial propagation is another design degree of freedom. If the two are independently defined, having the filter estimating error quantities, the set-up is known as indirect (or error-state) filtering. The direct (or total-state) filtering is achieved by merging the inertial algorithm and the filter propagation step. The resulting filter estimates total kinematics quantities, instead of errors or corrections. Although architecturally distinct the performances of these two set-ups can be made virtually equivalent [9]. Differences in behaviour may still arise from the way the corrections are performed, as observed by Wendel et al. [26]. The direct filtering scheme is, in general, more computationally intensive [26] and offers less design flexibility. Furthermore, as Steffes [21] shows, the indirect filtering architecture provides a simple yet powerful way to deal with measurement latency in the real-time implementation. 


\section{Time-differenced Integrated Carrier Phases}

The integrated carrier phase quantity is obtained within the GPS receiver through the accumulation of the phase increments from the carrier tracking loop. It is directly proportional to the range between receiver and emitting satellite, being by far the GPS raw measurement with the lowest noise level [10]. However, the initial phase value is unknown, preventing direct usage of this observable. Although this ambiguity term can be estimated using, for instance, ambiguity resolution/fixing methods in stand-alone or differential operation, allowing the integrated carrier measurement to be used as a range observable, this is not always possible or convenient.

A way to use these measurements without explicitly computing the ambiguities, is by forming Time-Differences of the integrated Carrier Phase (TDCP) measurements. Being a constant term, it is effectively cancelled out. The result is proportional to delta-range, i.e. a measure of displacement (or average velocity) rather than of range. The dual-epoch nature of the delta-range measurements makes their use in Kalman filtering more complicated than the usual single-epoch measurement. Nevertheless, several methods have been successfully used in [27, 30]. In Section 4 one of these methods will be adapted to the navigation filter at hand.

For channel $i$ at epoch $k$ the TDCP is formed as

$$
\begin{aligned}
\lambda \Delta \tilde{\varphi}_{i, k} & =\lambda\left(\tilde{\varphi}_{i, k}-\tilde{\varphi}_{i, k-1}\right)=\left(\rho_{i, k}+\lambda \mathrm{N}_{i}+c \tau_{i, k}\right)-\left(\rho_{i, k-1}+\lambda \mathrm{N}_{i}+c \tau_{i, k-1}\right) \\
& =\Delta \rho_{i, k}+c \Delta \tau_{i, k}
\end{aligned}
$$

where $\lambda$ is the carrier wave-length and $c$ the speed of light in vacuum. Note that the phase ambiguity $\mathrm{N}_{i}$ vanishes. $\Delta \tau$ gathers the differences of the remaining (timing) errors as

$$
\tau_{i, k}=\tau_{\mathrm{r}, k}+\tau_{\mathrm{s}, i, k}+\tau_{\mathrm{T}, k, i}+\tau_{\mathrm{I}, k, i}+\tau_{\mathrm{M}, k, i}+v_{k, i}
$$

where $\tau_{\mathrm{r}}$ and $\tau_{\mathrm{s}, i}$ are, respectively, the receiver and satellite clock offsets; $\tau_{\mathrm{T}, i}$ and $\tau_{\mathrm{I}, i}$ are tropospheric and ionospheric delays; $\tau_{\mathrm{M}, i}$ is the delay caused by signal reflection and multi-path; and $v_{\rho, i}$ is measurement noise.

Satellite clock error is (mostly) removed with information from the navigation message, while multipath delta-error is generally of impulse nature, occurring in reflective conditions (e.g. on the launch pad). The profile and effect of the receiver clock error and tropospheric delay are discussed in the following points.

\section{Receiver clock and common-mode error}

The common mode error is one that affects all channels equally. This is generally attributed to the receiver clock which is often modelled as a second order system [5] as

$$
\left[\begin{array}{c}
\dot{\tau}_{r} \\
\ddot{\tau}_{r}
\end{array}\right]=\left[\begin{array}{ll}
0 & 1 \\
0 & 0
\end{array}\right]\left[\begin{array}{l}
\tau_{r} \\
\dot{\tau}_{r}
\end{array}\right]+\mathbf{w}_{\tau_{r}},
$$

where $\mathbf{w}_{\tau_{r}} \sim N\left(\mathbf{0}, \mathbf{Q}_{\tau_{r}}\right)$. This system is then discretised, with noise model obtained, for instance, through an Allan variance fit as described in [3]. 


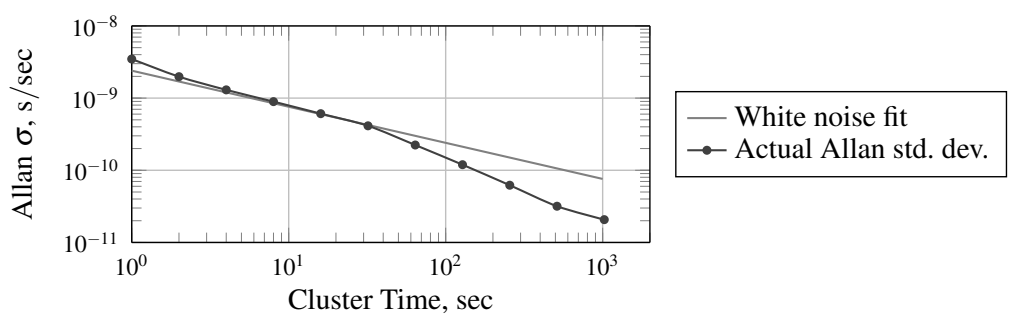

Fig. 2 Clock frequency error Allan std. dev. for a DLR Phoenix-HD receiver.

The receiver used in the present study, a DLR Phoenix-HD unit, uses the same internal clock model to correct both pseudorange and integrated carrier phase measurements. The TDCP common-mode error can be seen as an average clock frequency error over the differencing interval $\Delta t$ (here 1s), i.e.

$$
\Delta \tau_{r, k}=\tau_{r, k}-\tau_{r, k-1} \approx \Delta t \dot{\tau}_{r, k} .
$$

Fig. 2 shows the Allan standard deviation of the clock frequency error of a DLR Phoenix-HD receiver during a static test. This was obtained from pseudoranges preprocessed to extract the remaining errors. It is clear that the frequency error is dominated by white noise, leading the clock phase (or bias) to approximately behave as random walk. The receiver clock model used in the navigation filter can then be

$$
\begin{aligned}
& \text { Clock-bias state process: } & \tau_{\mathrm{r}, k+1} & =\tau_{\mathrm{r}, k}+\mathrm{w}_{\tau_{\mathrm{r}}, k}, \\
& \text { TDCP measurement: } & \mathbf{y}_{k} & =\boldsymbol{h}_{k}\left(\mathbf{x}_{k}\right)+\mathbf{1} c \Delta \tau_{\mathrm{r}, k}+\boldsymbol{v}_{k},
\end{aligned}
$$

$$
\text { with } \quad \mathrm{w}_{\tau_{\mathrm{r}}, k} \sim N\left(0, \sigma_{\tau_{\mathrm{r}}, k}^{2}\right), \quad \Delta \tau_{\mathrm{r}, k} \sim N\left(0, \sigma_{\Delta \tau_{\mathrm{r}, k}}^{2}\right), \quad \boldsymbol{v}_{k} \sim N\left(\mathbf{0}, \mathbf{R}_{v, k}\right),
$$

and

$$
E\left\{\mathrm{w}_{\tau_{\mathrm{r}, k}} \Delta \tau_{\mathrm{r}, k+1}\right\}=\sigma_{\tau_{\mathrm{r}}, k} \sigma_{\Delta \tau_{\mathrm{r}}, k+1}=\sigma_{\tau_{\mathrm{r}}, k}^{2}=\sigma_{\Delta \tau_{\mathrm{r}}, k}^{2} .
$$

In eq. (6), $\mathbf{1}$ is a column vector of ones. The correlation (8) between clock process noise and TDCP common-mode error will be taken into account upon derivation of the navigation filter. Note, that the fact that the clock drift is modelled as white noise avoids the need for one extra filter state, easing computational requirements.

\section{Tropospheric delay effect}

Refraction of GNSS signals in the Troposphere introduces a delay that depends on signal travel path and atmospheric conditions. For a land-based receiver this delay is fairly constant, affecting mostly position estimation. In a rocket application, the most important effect is not in position (though it is still affected) but in velocity. In fact, the ascending rocket motion through the atmosphere makes the tropospheric delay on each channel change quickly, yielding a hump-like error in rangerate (or delta-range), i.e. in velocity information. While for pseudorange (PR) and 


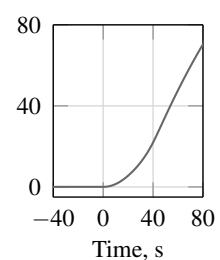

(a) Altitude, $\mathrm{km}$
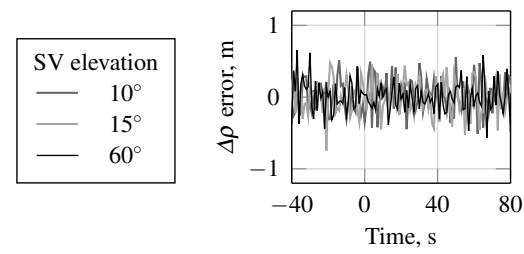

(e) Corrected Delta-PR

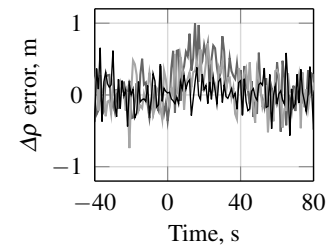

(b) Delta-PR

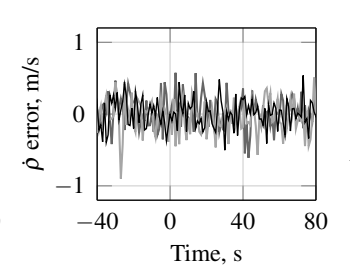

(f) Corrected PR-rate

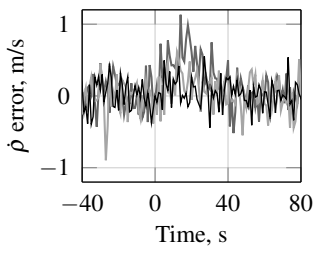

(c) PR-rate

Fig. 3 Error in Delta-PR, PR-rate and TDCP (at 1Hz) during rocket ascent, with and without tropospheric correction (see model in Appendix). Lift-off at $t=0 \mathrm{~s}$.

pseudorange-rate (PR-rate) this effect is mostly buried in measurement noise, for TDCP the considerably lower (single-channel) noise renders this effect flagrant. Fig. 3b-d show this effect on such observables (from SVs at different elevations) measured with a DLR Phoenix-HD GPS receiver stimulated by a GSS7700 SPIRENT GNSS emulator, which runs a NATO STANAG troposphere model [16]. The altitude profile followed is shown in Fig. $3 \mathrm{a}$.

The tropospheric delay-rate should be corrected or accounted for in filter tuning, otherwise the error in velocity estimation may surpass covariance bounds at this stage. This can worsen strapdown divergence in case of signal outage, which is rather likely during this flight leg given the violent dynamic events that may then occur. Many algorithms exists for tropospheric delay compensation. Most make use of atmospheric data either measured on-site or looked-up from tables of average regional/global values $[12,17]$. However, even with such correction the residual error in TDCP may still be considerably higher than the measurement noise level (Fig. $3 e-g)$. Therefore, for filter robustness, the measurement error covariance should account for the presence of such tropospheric residual.

Assume that the true tropospheric delay and delta-delay in channel $i$ can be given in terms of the modelled quantities as ${ }^{1}$

$$
\begin{aligned}
\tau_{\mathrm{T}, i, k} & =\left(1+\mathrm{s}_{\mathrm{T}}\right) h_{\tau_{\mathrm{T}}, i, k}\left(\mathbf{x}_{k}\right) \\
\Delta \tau_{\mathrm{T}, i, k} & =\left(1+\mathrm{s}_{\mathrm{T}}\right) \Delta h_{\tau_{\mathrm{T}}, i, k}\left(\mathbf{x}_{k}\right)=\left(1+\mathrm{s}_{\mathrm{T}}\right)\left(h_{\tau_{\mathrm{T}}, i, k}\left(\mathbf{x}_{k}\right)-h_{\tau_{\mathrm{T}}, i, k-1}\left(\mathbf{x}_{k}\right)\right),
\end{aligned}
$$

where $\mathrm{s}_{\mathrm{T}} \sim N\left(0, \sigma_{\mathrm{s}_{\mathrm{T}}}^{2}\right)$ is a constant unknown scale factor. $h_{\tau_{\mathrm{T}}, i, k}\left(\mathbf{x}_{k}\right)$ is the tropospheric delay correction model.

\footnotetext{
${ }^{1}$ As it shall be seen, the state vector of the navigation filter described in Section 4 allows $\Delta \tau_{\mathrm{T}, k}$ to be given only in terms of $\mathbf{x}_{k}$, needing not $\mathbf{x}_{k-1}$.
} 
The covariance of the post-correction residual vectors, $\boldsymbol{\delta} \tau_{\mathrm{T}, k}$ and $\boldsymbol{\delta} \boldsymbol{\Delta} \boldsymbol{\tau}_{\mathrm{T}, k}$, is

$$
\begin{aligned}
E\left\{\boldsymbol{\delta} \boldsymbol{\tau}_{\mathrm{T}, k} \boldsymbol{\delta} \boldsymbol{\tau}_{\mathrm{T}, k}^{\top}\right\} & =E\left\{\left(\boldsymbol{\tau}_{\mathrm{T}, k}-\hat{\boldsymbol{\tau}}_{\mathrm{T}, k}\right)\left(\boldsymbol{\tau}_{\mathrm{T}, k}-\hat{\boldsymbol{\tau}}_{\mathrm{T}, k}\right)^{\top}\right\} \\
& =\sigma_{\mathrm{s}_{\mathrm{T}}}^{2} \boldsymbol{h}_{\tau_{\mathrm{T}}, k}\left(\hat{\mathbf{x}}_{k}\right) \boldsymbol{h}_{\tau_{\mathrm{T}}, k}\left(\hat{\mathbf{x}}_{k}\right)^{\top}+\mathbf{H}_{\tau_{\mathrm{T}}, k} \mathbf{P}_{k} \mathbf{H}_{\tau_{\mathrm{T}}, k}^{\top},
\end{aligned}
$$

and

$$
\begin{aligned}
E\left\{\boldsymbol{\delta} \boldsymbol{\Delta} \boldsymbol{\tau}_{\mathrm{T}, k} \boldsymbol{\delta} \boldsymbol{\Delta} \boldsymbol{\tau}_{\mathrm{T}, k}^{\top}\right\} & =E\left\{\left(\boldsymbol{\Delta} \boldsymbol{\tau}_{\mathrm{T}, k}-\boldsymbol{\Delta} \hat{\boldsymbol{\tau}}_{\mathrm{T}, k}\right)\left(\boldsymbol{\Delta} \boldsymbol{\tau}_{\mathrm{T}, k}-\boldsymbol{\Delta} \hat{\boldsymbol{\tau}}_{\mathrm{T}, k}\right)^{\top}\right\} \\
& =\sigma_{\mathrm{s}_{\mathrm{T}}}^{2} \boldsymbol{\Delta} \boldsymbol{h}_{\tau_{\mathrm{T}}, k}\left(\hat{\mathbf{x}}_{k}\right) \boldsymbol{\Delta} \boldsymbol{h}_{\tau_{\mathrm{T}}, k}\left(\hat{\mathbf{x}}_{k}\right)^{\top}+\mathbf{H}_{\Delta \tau_{\mathrm{T}, k}, \mathbf{P}_{k} \mathbf{H}_{\Delta \tau_{\mathrm{T}}, k}^{\top},}
\end{aligned}
$$

with $\quad \Delta \boldsymbol{h}_{\tau_{\mathrm{T}}, k}\left(\hat{\mathbf{x}}_{k}\right)=\boldsymbol{h}_{\tau_{\mathrm{T}}, k}\left(\hat{\mathbf{x}}_{k}\right)-\boldsymbol{h}_{\tau_{\mathrm{T}}, k-1}\left(\hat{\mathbf{x}}_{k}\right), \quad \mathbf{H}_{\Delta \tau_{\mathrm{T}}, k}=\mathbf{H}_{\tau_{\mathrm{T}}, k}-\mathbf{H}_{\tau_{\mathrm{T}}, k-1}$,

and

$$
\mathbf{H}_{\tau_{\mathrm{T}}, k}=\left.\frac{\partial \boldsymbol{h}_{\tau_{\mathrm{T}}, k}\left(\mathbf{x}_{k}\right)}{\partial \mathbf{x}_{k}}\right|_{\hat{\mathbf{x}}_{k}}, \quad \mathbf{H}_{\tau_{\mathrm{T}}, k-1}=\left.\frac{\partial \boldsymbol{h}_{\tau_{\mathrm{T}}, k-1}\left(\mathbf{x}_{k}\right)}{\partial \mathbf{x}_{k}}\right|_{\hat{\mathbf{x}}_{k}} .
$$

$\mathbf{P}_{k}$ is the filter state estimate covariance matrix. The value of $\sigma_{s_{T}}$ is selected through simulation or flight data analysis, according to the performance of the correction algorithm in use, to make the filter is robust against the delay residuals. The tropospheric delay correction model used in this study can be found in Appendix.

\section{Hybrid Navigation Algorithm Design}

A diagram of the hybrid navigation system here developed is shown in Fig. 4. The system is tightly coupled with closed-loop indirect filtering (see Section 2). The IMU measurements are propagated at high-rate $(400 \mathrm{~Hz})$ by the strapdown routine while the raw GPS outputs (pseudoranges and integrated carrier phases), received at much lower rate $(1 \mathrm{~Hz})$, are used to update an error-state Kalman filter (running at $1 \mathrm{~Hz}$ ). The estimated state corrections, including IMU error terms, are fedback to the strapdown routine. As explained in the preceding section, a tropospheric delay model corrects the GPS measurements and is used for the measurement update noise model, as means of rendering the filter robust against correction residuals.

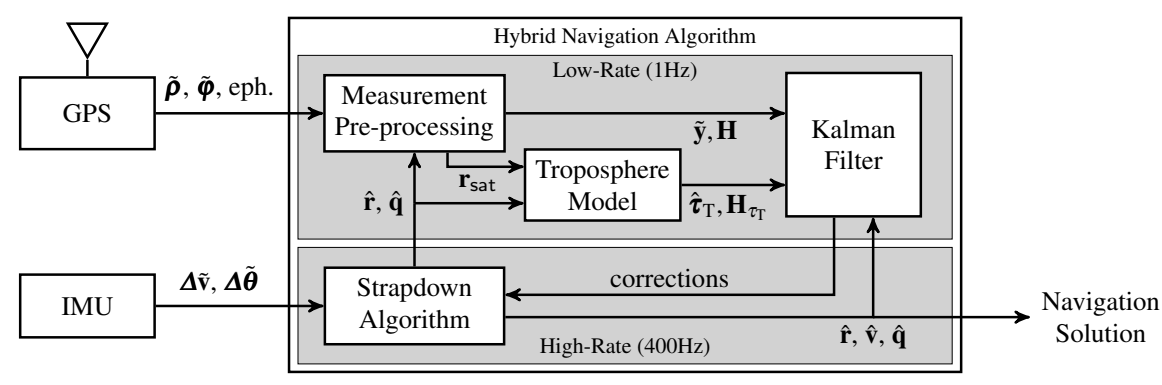

Fig. 4 Navigation algorithm block diagram. 


\subsection{Reference Frames and Time Indexing}

The WGS84 (World Geodetic System 1984) Earth-Centered Earth-Fixed (ECEF) is here denoted $E$; an Inertial reference frame, used to support the definition of absolute physical quantities, is denoted $I$; and a Body-fixed frame, with origin in the center of the IMU, is written $B$.

The time index of the high-rate tasks is denoted $j$, whereas that of the low-rate ones is $k$. The high-rate index is reset at each low-rate step, $t_{k}=t_{j=0}$, being $t_{k+1}=$ $t_{j=N}$, with $N=400$.

\subsection{Strapdown Propagation}

The vehicle body attitude with respect to ECEF frame is represented using the quaternion $\mathbf{q}_{B}^{E}$. This quantity is propagated according to

$$
\mathbf{q}_{B_{j+1}}^{E_{j+1}}=\left[\begin{array}{c}
\left(-\frac{1}{2} \mathbf{I}+\frac{1}{48}\left\|\boldsymbol{\Delta} \boldsymbol{\theta}_{j}^{B}\right\|^{2} \mathbf{I}+\frac{1}{24}\left[\boldsymbol{\Delta} \boldsymbol{\theta}_{j-1}^{B} \times\right]\right) \boldsymbol{\Delta} \boldsymbol{\theta}_{j}^{B} \\
1-\frac{1}{8}\left\|\boldsymbol{\Delta} \boldsymbol{\theta}_{j}^{B}\right\|^{2}
\end{array}\right] \mathbf{q}_{B_{j}}^{E_{j}} \mathbf{q}_{E_{j}}^{E_{j+1}}
$$

where the transition quaternion from $B_{j}$ to $B_{j+1}$ follows the third order algorithm by McKern [14]. $\left[\boldsymbol{\Delta} \boldsymbol{\theta}_{j-1}^{B} \times\right]$ is the skew-symmetric matrix of $\boldsymbol{\Delta}_{j-1}^{B}$.

The rigid-body translational kinematics can be given in inertial frame as

$$
\begin{aligned}
& \mathbf{v}_{j+1}^{I}=\mathbf{v}_{j}^{I}+\int_{t_{j}}^{t_{j+1}}\left(\mathbf{C}_{B}^{I} \mathbf{a}_{\mathrm{sf}}^{B}+\mathbf{g}^{I}\right) \mathrm{d} t=\mathbf{v}_{j}^{I}+\Delta \mathbf{v}_{\mathrm{sf}, j}^{I}+\Delta \mathbf{v}_{\mathrm{g}, j}^{I} \\
& \mathbf{r}_{j+1}^{I}=\mathbf{r}_{j}^{I}+\int_{t_{j}}^{t_{j+1}} \mathbf{v}^{I} \mathrm{~d} t=\mathbf{r}_{j}^{I}+\frac{\Delta t_{j}}{2}\left(\mathbf{v}_{j}^{I}+\mathbf{v}_{j+1}^{I}\right)
\end{aligned}
$$

where $\mathbf{g}^{I}$ and $\mathbf{a}_{\mathrm{sf}}^{B}$ are gravity and specific-force accelerations, respectively. The integral in (17) was solved using a trapezoidal method, with $\Delta t_{j}=t_{j+1}-t_{j}$. System (16-17) is easily transformed to ECEF yielding the propagation laws for $\mathbf{v}_{j}^{E}$ and $\mathbf{r}_{j}^{E}$.

The gravity induced Delta- $\mathrm{V}, \Delta \mathbf{v}_{\mathrm{g}, j}^{I}$, can be approximated evaluating the gravity field model at the midpoint of the integration period as

$$
\Delta \mathbf{v}_{\mathrm{g}, j}^{I} \approx \Delta t_{j} \mathbf{C}_{E_{j+\frac{1}{2}}}^{I} \mathbf{g}_{j+\frac{1}{2}}^{E}=\Delta t_{j} \mathbf{C}_{E_{j+\frac{1}{2}}^{I}}^{\mathbf{g}^{E}}\left(\mathbf{r}_{j+\frac{1}{2}}^{E}\right)
$$

where $\boldsymbol{g}^{E}\left(\mathbf{r}^{E}\right)$ is the ECEF gravity model function. The specific force Delta-V, $\Delta \mathbf{v}_{\mathrm{sf}, j}^{I}$, is also assumed to be taken at the midpoint of the integration period to account for the rotation of Body frame during this interval. It is given by

$$
\Delta \mathbf{v}_{\mathrm{sf}, j}^{I} \approx \mathbf{C}_{E_{j}}^{I} \mathbf{C}_{B_{j}}^{E_{j}} \mathbf{C}_{B_{j+\frac{1}{2}}^{B_{j}}} \Delta \mathbf{v}_{j}^{B}
$$

where $\Delta \mathbf{v}_{j}^{B}$ is the accelerometer measurement from $t_{j}$ to $t_{j+1}$. 


\subsection{Kalman Filter Equations}

The error-state filter equations from [22] are here modified to accommodate the correlation between measurement and process noise. Recall from Section 3 that, in the present system, the clock error in the TDCP is perfectly correlated with the driving noise of the clock-bias state.

The whole-state system and measurement models are written as

$$
\begin{aligned}
\mathbf{x}_{k+1} & =\boldsymbol{\phi}_{k+1 \mid k}\left(\mathbf{x}_{k}\right)+\mathbf{w}_{k} \\
\mathbf{y}_{k} & =\boldsymbol{h}_{k}\left(\mathbf{x}_{k}\right)+\boldsymbol{\varepsilon}_{k}+\boldsymbol{v}_{k},
\end{aligned}
$$

where $\mathbf{w}_{k} \sim N\left(\mathbf{0}, \mathbf{Q}_{k+1 \mid k}\right)$ is the process noise, and $\boldsymbol{\varepsilon}_{k} \sim N\left(\mathbf{0}, \mathbf{R}_{\varepsilon, k}\right)$ and $\boldsymbol{v}_{k} \sim$ $N\left(\mathbf{0}, \mathbf{R}_{v, k}\right)$ are measurement noises. $\boldsymbol{\varepsilon}$ is correlated to $\mathbf{w}$ according to

$$
E\left\{\boldsymbol{\varepsilon}_{k} \mathbf{w}_{k-1}^{\top}\right\}=\mathbf{B}_{k}^{\top} .
$$

The error-state system and measurement models are given, respectively, by

$$
\begin{aligned}
\boldsymbol{\delta} \mathbf{x}_{k+1} & =\mathbf{x}_{k+1}-\hat{\mathbf{x}}_{k+1}=\boldsymbol{\phi}_{k+1 \mid k}\left(\hat{\mathbf{x}}_{k}+\boldsymbol{\delta} \mathbf{x}_{k}\right)-\boldsymbol{\phi}_{k+1 \mid k}\left(\hat{\mathbf{x}}_{k}\right)+\mathbf{w}_{k} \\
\boldsymbol{\delta} \mathbf{y}_{k} & =\mathbf{y}_{k}-\hat{\mathbf{y}}_{k}=\boldsymbol{h}_{k}\left(\hat{\mathbf{x}}_{k}+\boldsymbol{\delta} \mathbf{x}_{k}\right)-\boldsymbol{h}_{k}\left(\hat{\mathbf{x}}_{k}\right)+\boldsymbol{\varepsilon}_{k}+\boldsymbol{v}_{k} .
\end{aligned}
$$

The covariance of the a priori error-state follows ${ }^{2}$

$$
\mathbf{P}_{(k+1)^{-}} \approx \boldsymbol{\Phi}_{k+1 \mid k} \mathbf{P}_{k^{+}} \boldsymbol{\Phi}_{k+1 \mid k}^{\top}+\mathbf{Q}_{k+1 \mid k}^{\top}
$$

where $\boldsymbol{\Phi}_{k+1 \mid k}$ is the Jacobian matrix of the vector function $\boldsymbol{\phi}_{k+1 \mid k}\left(\mathbf{x}_{k}\right)$ evaluated at the a posteriori whole-state estimate at time step $k, \hat{\mathbf{x}}_{k^{+}}$.

The filter correction step is given by

$$
\boldsymbol{\delta} \hat{\mathbf{x}}_{k^{+}}=\boldsymbol{\delta} \hat{\mathbf{x}}_{k^{-}}+\mathbf{K}_{k}\left(\boldsymbol{\delta} \mathbf{y}-\boldsymbol{h}_{k}\left(\hat{\mathbf{x}}_{k^{-}}+\boldsymbol{\delta} \hat{\mathbf{x}}_{k^{-}}\right)+\boldsymbol{h}_{k}\left(\hat{\mathbf{x}}_{k^{-}}\right)\right) .
$$

with the Kalman gain $\mathbf{K}_{k}$ given as in [7] as

$$
\mathbf{K}_{k}=\left(\mathbf{P}_{k^{-}} \mathbf{H}_{k}^{\top}+\mathbf{B}_{k}\right)\left(\mathbf{H}_{k} \mathbf{P}_{k^{-}} \mathbf{H}_{k}^{\top}+\mathbf{H}_{k} \mathbf{B}_{k}+\mathbf{B}_{k}^{\top} \mathbf{H}_{k}^{\top}+\mathbf{R}_{\varepsilon, k}+\mathbf{R}_{v, k}\right)^{-1}
$$

yielding

$$
\mathbf{P}_{k^{+}}=\left(\mathbf{I}-\mathbf{K}_{k} \mathbf{H}_{k}\right) \mathbf{P}_{k^{-}}-\mathbf{K}_{k} \mathbf{B}_{k}^{\top},
$$

where $\mathbf{H}_{k}$ is the Jacobian matrix of the measurement vector function $\boldsymbol{h}_{k}\left(\mathbf{x}_{k}\right)$ evaluated at the a priori whole-state estimate, $\hat{\mathbf{x}}_{k^{-}}$.

After a measurement update cycle the whole state is updated with the error-state vector, being the latter reset,

$$
\hat{\mathbf{x}}_{k^{+}}=\hat{\mathbf{x}}_{k^{-}}+\boldsymbol{\delta} \hat{\mathbf{x}}_{k^{+}}, \quad \boldsymbol{\delta} \hat{\mathbf{x}}_{k^{+}} \leftarrow \mathbf{0}
$$

\footnotetext{
2 a priori and a posteriori estimates are denoted, respectively, by the index superscripts - and + .
} 


\subsection{State Vector}

As stated in the previous point the navigation system at hand uses two state vectors: whole state and error state vectors. For each estimated whole state there is a corresponding error state. States can also be split into: strapdown (kinematics) states, IMU states (bias and scale-factor states) and measurement model states, as

$$
\mathbf{x}_{k}=\left[\mathbf{x}_{\mathrm{kin}, k}^{\top} \mathbf{x}_{\mathrm{imu}, k}^{\top} \mathbf{x}_{\text {meas }, k}^{\top}\right]^{\top}
$$

In general the error states are simple additive disturbances of the whole-states as in (23). The attitude is an exception. The error-state is instead a 3-component angle as

$$
\left[\begin{array}{c}
\frac{1}{2} \boldsymbol{\delta} \boldsymbol{\theta}_{k}^{B} \\
1
\end{array}\right] \approx \mathbf{q}_{B_{k}}^{E_{k}}\left(\hat{\mathbf{q}}_{B_{k}}^{E_{k}}\right)^{-1} .
$$

The set of kinematics whole-states $\mathbf{x}_{\mathrm{kin}, k}$ includes $\mathbf{v}_{k}^{E}, \mathbf{r}_{k}^{E}$ and $\mathbf{q}_{B_{k}}^{E_{k}}$, being the corresponding error-states $\boldsymbol{\delta} \mathbf{v}_{k}^{E}, \boldsymbol{\delta} \mathbf{r}_{k}^{E}$ and $\boldsymbol{\delta} \boldsymbol{\theta}_{k}^{B}$. The remaining state sets, $\mathbf{x}_{\text {imu }}$ and $\mathbf{x}_{\text {meas }}$, are introduced in the following sections.

\subsection{IMU Error Model}

The accelerometer and gyroscope measurements were modelled including the disturbing effects of scale factor and bias errors, as

$$
\begin{aligned}
\boldsymbol{\Delta}_{\mathbf{v}_{\mathrm{sf}, j}^{B}}^{B} & =\left(\mathbf{I}+\operatorname{diag}\left(\mathbf{s}_{\mathrm{a}, j}^{B}\right)\right) \boldsymbol{\Delta} \mathbf{v}_{\mathrm{sf}, j}^{B}+\Delta t_{j} \mathbf{b}_{\mathrm{a}, j}^{B}+\boldsymbol{v}_{\mathrm{a}, j} \\
\boldsymbol{\Delta} \tilde{\boldsymbol{\theta}}_{j}^{B} & =\left(\mathbf{I}+\operatorname{diag}\left(\mathbf{s}_{\mathrm{g}, j}^{B}\right)\right) \boldsymbol{\Delta} \boldsymbol{\theta}_{j}^{B}+\Delta t_{j} \mathbf{b}_{\mathrm{g}, j}^{B}+\boldsymbol{v}_{\mathrm{g}, j} .
\end{aligned}
$$

where $\boldsymbol{\Delta} \tilde{\mathbf{v}}_{\mathrm{sf}, j}^{B}$ and $\boldsymbol{\Delta} \tilde{\boldsymbol{\theta}}_{j}^{B}$ are the measured increments, while $\boldsymbol{\Delta} \mathbf{v}_{\mathrm{sf}, j}^{B}$ and $\boldsymbol{\Delta} \boldsymbol{\theta}_{j}^{B}$ are the actual quantities. $\boldsymbol{v}_{\mathrm{a}, j}$ and $\boldsymbol{v}_{\mathrm{g}, j}$ are measurement noise terms. The bias $\mathbf{b}_{\mathrm{a}, j}^{B}$ and $\mathbf{b}_{\mathrm{g}, j}^{B}$, and scale-factors $\mathbf{b}_{\mathrm{a}, j}^{B}$ and $\mathbf{b}_{\mathrm{g}, j}^{B}$ are the filter states in $\mathbf{x}_{\mathrm{imu}, k}$. To each of these quantities corresponds an error-state: $\boldsymbol{\delta} \mathbf{b}_{\mathrm{a}, j}^{B}, \boldsymbol{\delta} \mathbf{b}_{\mathrm{g}, j}^{B}, \boldsymbol{\delta} \mathbf{s}_{\mathrm{a}, j}^{B}$, and $\boldsymbol{\delta} \mathbf{s}_{\mathrm{g}, j}^{B}$. These IMU states are modelled as random walk processes with random initial condition. The values used correspond to the fibre-optic gyros and servo accelerometers of the tactical grade IMU of the HNS version flown on-board of SHEFEX2 [22].

\subsection{Filter Propagation}

The strapdown algorithm presented earlier propagates the kinematics whole-states at high-rate (time-index $j$ ). Linearizing the error-state system

$$
\boldsymbol{\delta} \mathbf{x}_{j+1}=\boldsymbol{\phi}_{j+1 \mid j}\left(\hat{\mathbf{x}}_{k}+\boldsymbol{\delta} \mathbf{x}_{j}\right)-\boldsymbol{\phi}_{j+1 \mid j}\left(\hat{\mathbf{x}}_{j}\right)+\mathbf{w}_{j} \approx \boldsymbol{\Phi}_{j+1 \mid j} \boldsymbol{\delta} \mathbf{x}_{j}+\mathbf{w}_{j},
$$


yielding

$$
\boldsymbol{\Phi}_{j+1 \mid j}=\left[\begin{array}{ccc}
\boldsymbol{\Phi}_{\text {kin }}^{\mathrm{kin}} & \boldsymbol{\Phi}_{\text {kin }}^{\mathrm{imu}} & \mathbf{0} \\
\mathbf{0} & \boldsymbol{\Phi}_{\text {imu }}^{\mathrm{imu}} & \mathbf{0} \\
\mathbf{0} & \mathbf{0} & \boldsymbol{\Phi}_{\text {meas }}^{\text {meas }}
\end{array}\right]_{j+1 \mid j}
$$

and assuming that the accumulated system noise over one low-rate step (index $k$ ) is lower than the total state uncertainty, the low-rate transition and process noise covariance matrices can be approximated as

$$
\boldsymbol{\Phi}_{k+1 \mid k} \approx \prod_{j=0}^{N-1} \boldsymbol{\Phi}_{j+1 \mid j}, \quad \mathbf{Q}_{k+1 \mid k} \approx \sum_{j=0}^{N-1} \mathbf{Q}_{j+1 \mid j}
$$

where $t_{k}=t_{j=0}$ and $t_{k+1}=t_{j=N}$.

The sub-matrices $\Phi_{\mathrm{kin}}^{\mathrm{kin}}$ and $\boldsymbol{\Phi}_{\mathrm{kin}}^{\mathrm{imu}}$ can be easily derived from the linearization of the translational kinematics (16-17), with (18-19), after transforming all to the ECEF frame; and the error-angle kinematics given in [22] by

$$
\boldsymbol{\delta} \boldsymbol{\theta}_{j+1}^{B}=\hat{\mathbf{C}}_{B_{j}}^{B_{j+1}} \boldsymbol{\delta} \boldsymbol{\theta}_{j}^{B}-\boldsymbol{\delta} \boldsymbol{\Delta} \boldsymbol{\theta}_{j}^{B}+\text { noise . }
$$

The mapping from IMU error-states $\boldsymbol{\delta} \mathbf{x}_{\text {imu }}$ to the error in the increments $\boldsymbol{\delta} \boldsymbol{\Delta} \mathbf{v}^{B}$ and $\boldsymbol{\delta} \boldsymbol{\Delta} \boldsymbol{\theta}^{B}$ is obtained through the (approximate) inverse of the IMU error model (32-33)

$$
\begin{aligned}
\boldsymbol{\delta} \boldsymbol{\Delta} \mathbf{v}_{j}^{B} & =-\operatorname{diag}\left(\boldsymbol{\Delta} \tilde{\mathbf{v}}_{j}^{B}\right) \boldsymbol{\delta} \mathbf{s}_{\mathrm{a}, j}^{B}-\Delta t_{j} \boldsymbol{\delta} \mathbf{b}_{\mathrm{a}, j}^{B}-\boldsymbol{v}_{\mathrm{a}, j} \\
\boldsymbol{\delta} \boldsymbol{\Delta} \boldsymbol{\theta}_{j}^{B} & =-\operatorname{diag}\left(\boldsymbol{\Delta} \tilde{\boldsymbol{\theta}}_{j}^{B}\right) \boldsymbol{\delta} \mathbf{s}_{\mathrm{g}, j}^{B}-\Delta t_{j} \boldsymbol{\delta} \mathbf{b}_{\mathrm{g}, j}^{B}-\boldsymbol{v}_{\mathrm{g}, j} .
\end{aligned}
$$

The IMU error random walk states mean that $\Phi_{\text {imu }}^{\mathrm{imu}}=\mathbf{I}$. As for $\Phi_{\text {meas }}^{\text {meas }}$, it shall be easily formed upon definition, in the next points, of the filter update models.

\subsection{Filter Updates}

The filter update models used for Pseudorange and Time-differenced Integrated Carrier Phase measurements are as follows.

\section{Pseudorange update}

The model for a set of pseudorange measurements be written

$$
\mathbf{y}_{\rho, k}=\boldsymbol{h}_{\rho, k}\left(\mathbf{x}_{k}\right)+\boldsymbol{v}_{\rho, k},
$$

where the measurement (vector) function $\boldsymbol{h}_{\rho}\left(\mathbf{x}_{k}\right)$ is for channel $i$ given by

$$
h_{\rho, i, k}\left(\mathbf{x}_{k}\right)=\rho_{i, k}\left(\mathbf{x}_{k}\right)+c\left(\tau_{\mathrm{r}, k}+\tau_{\mathrm{T}, i, k}\left(\mathbf{x}_{k}\right)\right)+\mathrm{b}_{\rho, i, k},
$$

with range 


$$
\rho_{i, k}\left(\mathbf{x}_{k}\right)=\left\|\mathbf{C}_{E\left(t_{s, i, k}\right)}^{E_{k}} \mathbf{r}_{i}^{E}\left(t_{\mathrm{s}, i, k}\right)-\mathbf{r}_{k}^{E}-\mathbf{C}_{B_{k}}^{E_{k}} k_{\text {a }}^{B}\right\| .
$$

The satellite position $\mathbf{r}_{i}^{E}$ is evaluated at the emitting instant $t_{\mathrm{s}, i, k}$ and translated to the ECEF coordinates at time of reception. $\mathbf{l}_{\mathbf{a}}$ is the lever-arm from IMU to GPS antenna. The pseudorange bias state $\mathrm{b}_{\rho, i}$ is modelled as a random constant plus random walk process (i.e. $\Phi_{\mathrm{b}_{\rho, i}}^{\mathrm{b}_{\rho, i}}=1$ ). The receiver clock-bias state $\tau_{\mathrm{r}}$ has the dynamics given by (6). The tropospheric delay $\tau_{\mathrm{T}, i, k}\left(\mathbf{x}_{k}\right)$ is given by (9).

The error-measurement model is similar to (24) as

$$
\boldsymbol{\delta}_{\rho, k}=\boldsymbol{h}_{\rho, k}\left(\hat{\mathbf{x}}_{k}+\boldsymbol{\delta} \mathbf{x}_{k}\right)-\boldsymbol{h}_{\rho, k}\left(\hat{\mathbf{x}}_{k}\right)+\boldsymbol{v}_{\rho, k} .
$$

where for the $i^{\text {th }}$ channel

$$
h_{\rho, i, k}\left(\hat{\mathbf{x}}_{k}+\boldsymbol{\delta} \mathbf{x}_{k}\right)-h_{\rho, i, k}\left(\hat{\mathbf{x}}_{k}\right)=\delta \rho_{i, k}\left(\boldsymbol{\delta} \mathbf{x}_{k}\right)+c\left(\delta \tau_{\mathrm{r}, k}+\delta \tau_{\mathrm{T}, i, k}\left(\boldsymbol{\delta} \mathbf{x}_{k}\right)\right)+\delta \mathrm{b}_{\rho, i, k},
$$

with

$$
\begin{aligned}
\delta \rho_{i, k}\left(\boldsymbol{\delta} \mathbf{x}_{k}\right) & \approx-\hat{\mathbf{e}}_{\rho, i, k}^{E}{ }^{\top}\left(\boldsymbol{\delta} \mathbf{r}_{k}^{E}+\hat{\mathbf{C}}_{B_{k}}^{E_{k}}\left[\mathbf{l}_{\mathrm{a}}^{B} \times\right] \boldsymbol{\delta} \boldsymbol{\theta}_{k}^{B}\right) \\
\delta \tau_{\mathrm{T}, i, k}\left(\boldsymbol{\delta} \mathbf{x}_{k}\right) & \approx \mathrm{s}_{\mathrm{T}, i} h_{\tau_{\mathrm{T}}, i, k}\left(\hat{\mathbf{x}}_{k}\right)+\mathbf{h}_{\tau_{\mathrm{T}}, i, k} \boldsymbol{\delta} \mathbf{x}_{k} .
\end{aligned}
$$

$\hat{\mathbf{e}}_{\rho, i, k}^{E}$ is the estimated unit range vector from receiver to satellite $i$, and $\mathbf{h}_{\tau_{\mathrm{T}}, i, k}$, as derived in Appendix, is the Jacobian row vector of the tropospheric delay model function $h_{\tau_{\mathrm{T}}, i, k}$ with respect to the error-state vector.

The covariance of the error-measurement (43) is

$$
E\left\{\boldsymbol{\delta} \mathbf{y}_{\rho, k} \boldsymbol{\delta} \mathbf{y}_{\rho, k}^{\top}\right\} \approx \mathbf{H}_{\rho, k} \mathbf{P}_{k} \mathbf{H}_{\rho, k}^{\top}+c^{2} \sigma_{\mathrm{S}_{\mathrm{T}}}^{2} \boldsymbol{h}_{\tau_{\mathrm{T}}, k}\left(\hat{\mathbf{x}}_{k}\right) \boldsymbol{h}_{\tau_{\mathrm{T}}, k}\left(\hat{\mathbf{x}}_{k}\right)^{\top}+\mathbf{R}_{\rho, k},
$$

where

$$
\mathbf{H}_{\rho, k}=\left[\mathbf{h}_{\rho, 1, k}^{\top} \cdots \mathbf{h}_{\rho, i, k}^{\top} \cdots \mathbf{h}_{\rho, n, k}^{\top}\right]^{\top},
$$

with non-null partial derivatives

$$
\begin{array}{ll}
\mathbf{h}_{\rho, i, k}^{\mathrm{r}}=-\hat{\mathbf{e}}_{\rho, i, k}^{E}+c \mathbf{h}_{\tau_{\mathrm{T}}, i}^{\mathrm{r}}, & \mathbf{h}_{\rho, i, k}^{\theta}=-\hat{\mathbf{e}}_{\rho, i, k}^{E} \hat{\mathbf{C}}_{B_{k}}^{E_{k}}\left[\mathbf{l}_{\mathrm{a}}^{B} \times\right]+c \mathbf{h}_{\tau_{\mathrm{T}}, i}^{\theta}, \\
\mathbf{h}_{\rho, i, k}^{\tau_{r}}=c, & \mathrm{~h}_{\rho, i, k}^{\mathrm{b}_{\rho, i}}=1 .
\end{array}
$$

$\mathbf{h}_{\tau_{\mathrm{T}}, i, k}^{\mathrm{r}}$ and $\mathbf{h}_{\tau_{\mathrm{T}}, i, k}^{\theta}$ are the components of $\mathbf{h}_{\tau_{\mathrm{T}}, i, k}$ corresponding to $\boldsymbol{\delta} \mathbf{r}_{k}^{E}$ and $\boldsymbol{\delta} \boldsymbol{\theta}_{k}^{B}$.

\section{Time-differenced Integrated Carrier Phase update}

The TDCP measurement set can be modelled in the form

$$
\mathbf{y}_{\Delta \rho, k}=\boldsymbol{h}_{\Delta \rho, k}\left(\mathbf{x}_{k}\right)+\mathbf{1} c \Delta \tau_{\mathrm{r}, k}+\boldsymbol{v}_{\Delta \rho, k},
$$

where $\boldsymbol{v}_{\Delta \rho, k} \sim N\left(\mathbf{0}, \mathbf{R}_{\Delta \rho, k}\right)$ holds the single-channel noises and $\Delta \tau_{\mathrm{r}, k} \sim N\left(0, \sigma_{\Delta \tau_{\mathrm{r}}, k}^{2}\right)$, the TDCP clock error in (6), is perfectly correlated to the process noise of $\tau_{\mathrm{r}}$, as (8). 
The state-to-measurement mapping function is for channel $i$

$$
h_{\Delta \rho, i, k}\left(\mathbf{x}_{k}\right)=\rho_{i, k}\left(\mathbf{x}_{k}\right)-\rho_{i, k-1}\left(\mathbf{x}_{k}\right)+c \Delta \tau_{\mathrm{T}, i, k}\left(\mathbf{x}_{k}\right),
$$

where $\rho_{i, k}\left(\mathbf{x}_{k}\right)$ is as (42), $\Delta \tau_{\mathrm{T}, i, k}\left(\mathbf{x}_{k}\right)$ follows (10), and

$$
\rho_{i, k-1}\left(\mathbf{x}_{k}\right)=\left\|\mathbf{C}_{E\left(t_{\mathrm{s}, i, k-1}\right)}^{E_{k-1}} \mathbf{r}_{i}^{E}\left(t_{\mathrm{s}, i, k-1}\right)-\mathbf{r}_{\mathrm{a}, k-1, k}^{E_{k-1}}\right\|
$$

$\mathbf{r}_{\mathrm{a}, k-1, k}^{E_{k-1}}$ is a delayed state of GPS antenna position. This is a constant state, with null process noise, that is reset after each measurement update cycle as

$$
\left\{\hat{\mathbf{r}}_{\mathrm{a}, k-1}^{E_{k-1}}\right\}_{k^{+}} \leftarrow\left\{\hat{\mathbf{r}}^{E}+\hat{\mathbf{C}}_{B}^{E} \mathbf{l}_{\mathrm{a}}^{B}\right\}_{k^{+}}
$$

Note that $\mathbf{r}_{\mathrm{a}, k-1}^{E_{k-1}}$ is treated as a considered state, i.e. it is not updated ( $\left.\mathbf{K}_{k}^{\mathrm{r}_{\mathrm{a}}}=\mathbf{0}\right)$. This renders the computational effort required by the inclusion of this state relatively low. Upon reset its covariance and cross-covariance (to the remaining states) are set to

$$
\left\{\mathbf{P}_{\mathrm{xr}_{\mathrm{a}}}\right\}_{k^{+}} \leftarrow\left\{\mathbf{P}_{\mathrm{Xx}} \boldsymbol{\Phi}_{\mathrm{r}_{\mathrm{a}}, 0}^{\mathrm{x}}\right\}_{k^{+}}^{\top}, \quad\left\{\mathbf{P}_{\mathrm{r}_{\mathrm{a}} \mathrm{r}_{\mathrm{a}}}\right\}_{k^{+}} \leftarrow \mathbf{P}_{\mathrm{r}_{\mathrm{a}}, 0}
$$

where $\mathbf{P}_{\mathrm{xx}}$ is the covariance matrix of the remaining states, $\boldsymbol{\Phi}_{\mathrm{r}_{\mathrm{a}}, 0}$ is sparse with only

$$
\boldsymbol{\Phi}_{\mathrm{r}_{\mathrm{a}}, 0}^{\mathrm{r}}=\mathbf{I}, \quad \boldsymbol{\Phi}_{\mathrm{r}_{\mathrm{a}}, 0}^{\theta}=\hat{\mathbf{C}}_{B_{k}}^{E_{k}}\left[\mathbf{l}_{\mathrm{a}}^{B} \times\right],
$$

and $\mathbf{P}_{\mathrm{r}_{\mathrm{a}}, 0}$ is a small initial covariance used to avoid numerical problems.

The error-measurement $\boldsymbol{\delta} \mathbf{y}_{\Delta \rho, k}$ follows a similar logic to (43), as

$$
\boldsymbol{\delta}_{\Delta \rho, k}=\boldsymbol{h}_{\Delta \rho, k}\left(\hat{\mathbf{x}}_{k}+\boldsymbol{\delta} \mathbf{x}_{k}\right)-\boldsymbol{h}_{\Delta \rho, k}\left(\hat{\mathbf{x}}_{k}\right)+\mathbf{1} c \Delta \tau_{\mathrm{r}, k}+\boldsymbol{v}_{\Delta \rho, k}
$$

being for each channel $i$

and

$$
h_{\Delta \rho, i, k}\left(\hat{\mathbf{x}}_{k}+\boldsymbol{\delta} \mathbf{x}_{k}\right)-h_{\Delta \rho, i, k}\left(\hat{\mathbf{x}}_{k}\right)=\delta \Delta \rho_{i, k}\left(\boldsymbol{\delta} \mathbf{x}_{k}\right)+c \delta \Delta \tau_{\mathrm{T}, i, k}\left(\boldsymbol{\delta} \mathbf{x}_{k}\right)
$$

$$
\begin{gathered}
\delta \Delta \rho_{i, k}\left(\boldsymbol{\delta} \mathbf{x}_{k}\right) \approx \delta \rho_{i, k}\left(\boldsymbol{\delta} \mathbf{x}_{k}\right)+\hat{\mathbf{e}}_{\rho, i, k-1}^{E}{ }^{\top} \boldsymbol{\delta} \mathbf{r}_{\mathrm{a}, k-1}^{E_{k-1}} \\
\delta \Delta \tau_{\mathrm{T}, i, k}\left(\boldsymbol{\delta} \mathbf{x}_{k}\right) \approx \mathrm{s}_{\mathrm{T}, i} \Delta h_{\tau_{\mathrm{T}}, i, k}\left(\hat{\mathbf{x}}_{k}\right)+\mathbf{h}_{\tau_{\mathrm{T}}, i, k} \boldsymbol{\delta} \mathbf{x}_{k}-\mathbf{h}_{\tau_{\mathrm{T}}, i, k-1}^{\mathrm{r}} \boldsymbol{\delta} \mathbf{r}_{\mathrm{a}, k-1}^{E_{k-1}}
\end{gathered}
$$

where $\delta \rho_{i, k}\left(\boldsymbol{\delta} \mathbf{x}_{k}\right)$ is as (45).

The covariance of $\boldsymbol{\delta} \mathbf{y}_{\Delta \rho, k}$ is formed accounting for the correlation (8) as

$$
\begin{aligned}
E\left\{\boldsymbol{\delta}_{\Delta \rho, k} \boldsymbol{\delta} \mathbf{y}_{\Delta \rho, k}^{\top}\right\} \approx \mathbf{H}_{\Delta \rho, k} \mathbf{P}_{k} \mathbf{H}_{\Delta \rho, k}^{\top}+c^{2} \sigma_{\mathrm{s}_{\mathrm{T}}}^{2} \Delta \boldsymbol{h}_{\tau_{\mathrm{T}}, k}\left(\hat{\mathbf{x}}_{k}\right) \boldsymbol{\Delta} \boldsymbol{h}_{\tau_{\mathrm{T}}, k}\left(\hat{\mathbf{x}}_{k}\right)^{\top} \\
+3 c^{2} \sigma_{\Delta \tau_{\mathrm{r}, k}, \mathbf{1 1}^{\top}+\mathbf{R}_{\Delta \rho, k}}
\end{aligned}
$$

where

$$
\mathbf{H}_{\Delta \rho, k}=\left[\mathbf{h}_{\Delta \rho, 1, k}^{\top} \cdots \mathbf{h}_{\Delta \rho, i, k}^{\top} \cdots \mathbf{h}_{\Delta \rho, n, k}^{\top}\right]^{\top},
$$

with non-null components 


$$
\mathbf{h}_{\Delta \rho, i, k}^{\mathrm{r}}=\mathbf{h}_{\rho, i, k}^{\mathrm{r}}, \quad \mathbf{h}_{\Delta \rho, i, k}^{\theta}=\mathbf{h}_{\rho, i, k}^{\theta}, \quad \mathbf{h}_{\Delta \rho, i, k}^{\mathrm{r}_{\mathrm{a}}}=\hat{\mathbf{e}}_{\rho, i, k-1}^{E}{ }^{\top}-c \mathbf{h}_{\tau_{\mathrm{T}}, i, k-1}^{\mathrm{r}} .
$$

\section{Testing Results}

The navigation algorithm here presented was Hardware-in-the-Loop (HIL) tested using real GPS data. A SPIRENT GNSS emulator was used to stimulate a PhoenixHD receiver, having its output been filtered offline. Atmospheric disturbances such as Ionospheric and Tropospheric delays (STANAG tropospheric model [16]) were included in the emulation. The trajectory used follows a similar profile to that of the SHEFEX2 mission [24]. Fig. 5 shows the altitude time history of the two-burn sounding rocket flight. The IMU measurements were simulated using a tacticalgrade unit model with specifications as shown in Table 1.

\section{Tropospheric Delay Correction}

Velocity estimation performance during the atmospheric ascent is compared in Fig. 6 for four different set-ups using both TDCP and PR updates:

1) uncorrected measurements and update models neglecting Tropospheric delay;

2) uncorrected measurements but inflated measurement model noise values;

3) corrected measurements neglecting correction residuals; and

4) corrected measurements considering correction residuals derived in Section 3.

To compensate for the uncorrected atmospheric errors in the GPS signal, Steffes [23] used $1 \sigma$ covariance of the PR measurements scheduled in altitude as: $50 \mathrm{~m}$ for altitudes below $10 \mathrm{~km}$ (also accounting for multipath on the pad), $10 \mathrm{~m}$ for altitudes within $10-200 \mathrm{~km}$, and $2 \mathrm{~m}$ for altitudes above $200 \mathrm{~km}$. Configuration 2) employs these same values and a similar conservative schedule for the TDCP measurements.

The advantage of the configurations using corrected measurements is clear upon comparison of Fig. 6a and 6b. Note, however, that set-ups without covariance compensation, either against the entire tropospheric delay or the leftover correction residual, show signs of severe mismodelling, behaving over-optimistically. The proposed compensation method presented in Section 3 successfully maintains the filter coherence, further reducing the impact of the residual delay on the estimation.

\section{Nominal Performance}

In Fig. 7 the estimation error in terms of the kinematic states of the algorithm developed herein (filter 4 in the previous analysis) is compared to:

0 an uncorrected PR-only SHEFEX2 HNS-like configuration [23, 22]; and

$\circ$ a PR-only set-up with tropospheric corrections as described in Section 3. 


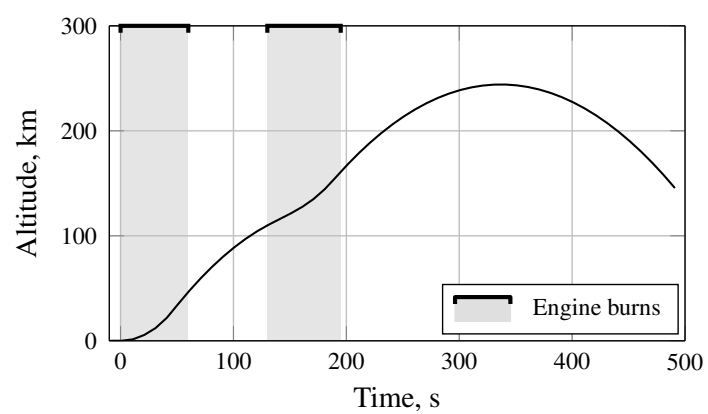

Fig. 5 Altitude profile of SHEFEX2 mission.

\begin{tabular}{lcc} 
& Gyroscope & Accelerometer \\
\hline Sensor range & $\pm 1000 \mathrm{deg} / \mathrm{s}$ & $\pm 5 \mathrm{~g}$ \\
Bandwidth & $500 \mathrm{~Hz}$ & $200 \mathrm{~Hz}$ \\
Axis misalignment & $0.5 \mathrm{mrad}$ & $0.5 \mathrm{mrad}$ \\
Angle/vel. random walk & $0.03 \mathrm{deg} / \sqrt{\mathrm{h}}$ & $50 \mu \mathrm{g} / \sqrt{\mathrm{Hz}}$ \\
Bias repeatability & $1 \mathrm{deg} / \mathrm{h}$ & $2 \mathrm{mg}$ \\
Bias instability & $0.03 \mathrm{deg} / \mathrm{h}$ & $50 \mu \mathrm{g}$ \\
Scale-factor repeatability & $300 \mathrm{ppm}$ & $1500 \mathrm{ppm}$ \\
\hline
\end{tabular}

Table 1 IMU (1 $\sigma)$ specifications [22].

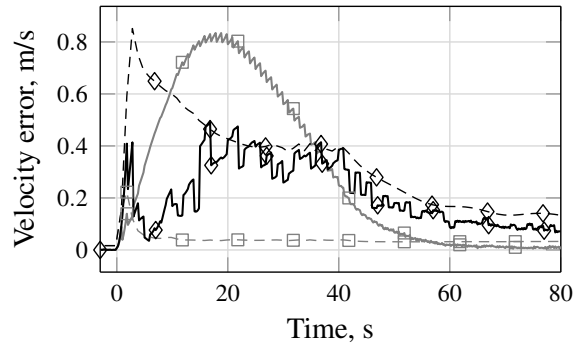

(a) Uncorrected TDCP + PR

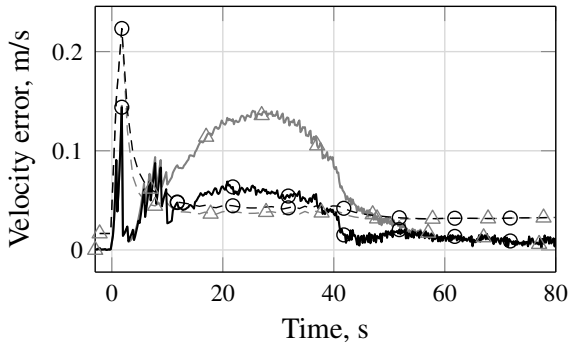

(b) Corrected TDCP + PR

\begin{tabular}{lccc}
\multicolumn{2}{r}{ Line Style $^{\mathrm{a}}$} & Tropo Corrected & Covariance Compensation \\
\hline 1$)$ & - & no & none \\
$2)$ & - & no & R scheduled $v s$ altitude as in [23] \\
$3)$ & - & yes & none \\
$4)$ & - & yes & as described in Section 3 \\
\hline \multicolumn{2}{c}{ a Dashed lines show $1 \sigma$ covariance bound. }
\end{tabular}

Fig. 6 Velocity estimation error with and without Tropospheric delay correction and covariance compensation. 
Once again, using tropospheric delay correction, even if only employing PR updates, considerably improves estimation during the atmospheric flight leg, especially in terms of velocity and attitude. However, it is the addition of TDCP measurements that yields the greatest improvement. As expected, given the delta-range nature of the TDCP observables and its low noise, velocity error sees the most significant reduction (about 10-fold throughout). Attitude estimation also benefits from the more accurate velocity information, especially during the more dynamic flight phases.

\section{GNSS Outage}

Vehicle dynamics can cause loss of lock in the GNSS tracking loops. Events such as separation, ignition, peak dynamic pressure, sonic transition are especially critical [11]. Fig. 8 compares the developed filter to the configurations described in the previous point under a $30-\mathrm{sec}$ outage starting at $t=20 \mathrm{~s}$. While all filters visibly drift, the proposed configuration clearly sees its velocity, position and attitude estimation diverge the least during the outage. The lower estimation error, granted especially by the use of TDCP updates, results in a better initial state for the pure inertial propagation during drop-out, thus yielding superior outage performance.

\section{Conclusion}

A tightly coupled, Time-differenced Carrier Phase and Pseudorange updated, hybrid navigation system has been presented. In the light of rocket launch conditions, the chosen architecture yields added robustness with respect to a loose coupling, while being realizable with COTS components, contrarily to the ultra-tight set-up. The receiver clock error dynamics affecting both TDCP and PR observables has been analysed for the receiver at hand and approximated by a single-state model, avoiding the wasteful cross-channel differences while carrying little extra computational effort. Tropospheric delta-delay has been found to be a major disturbance of the TDCP measurements. Although conservative measurement model tuning at lower atmosphere could render the filter coherent under this effect, estimation performance is poor when compared to a feed-forward corrected set-up. The residuals of this correction, however, still caused severe mismodelling. The proposed correction scheme with covariance compensation was shown to yield robustness against such residuals, maintaining filter coherence. The performance of the overall system has been compared to a SHEFEX2 HNS-like baseline configuration through HIL simulation, showing significant improvements in nominal and outage conditions. 


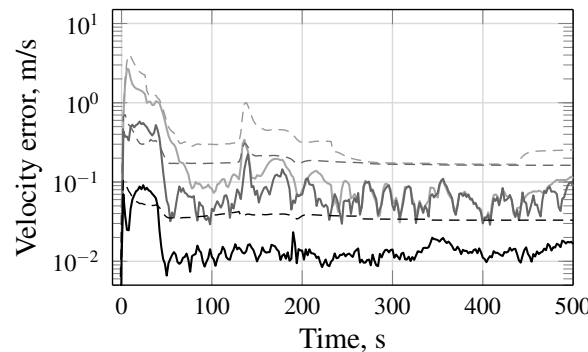

(a) Velocity error

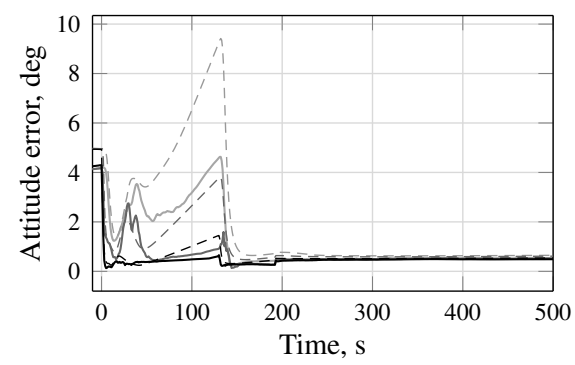

(c) Attitude error

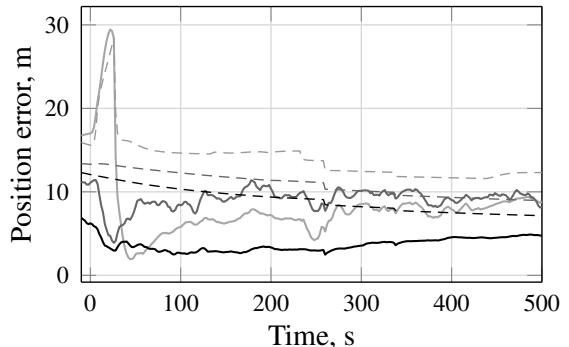

(b) Position error
— PR, uncorrected (SHEFEX2 HNS-like)

— PR, robust Tropo correction

- TDCP+PR, robust Tropo correction $1 \sigma$ covariance bounds as dashed lines

Fig. 7 Nominal estimation performance of the proposed system compared to two PR-only filters.

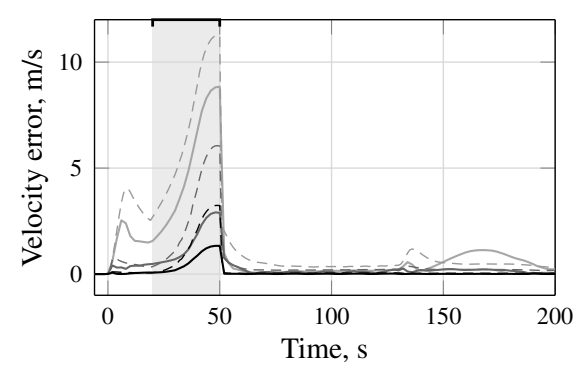

(a) Velocity error

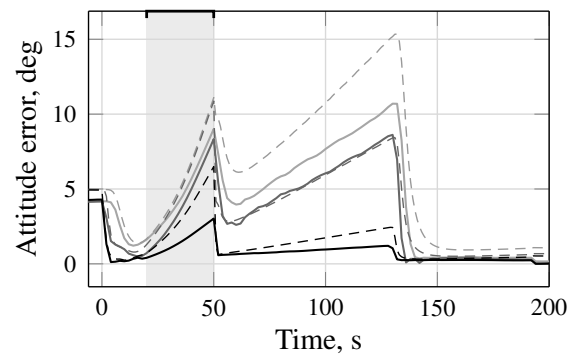

(c) Attitude error

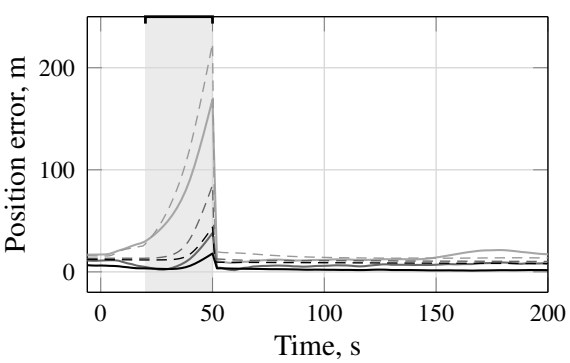

(b) Position error

Fig. 8 Estimation performance comparison in case of GNSS outage. 


\section{Appendix}

Parkinson et al. [17] suggest the following coarse tropospheric delay model, which does not require in situ atmospheric measurements nor the use of look-up tables:

$$
\begin{gathered}
h_{\tau_{\mathrm{T}}, i, k}\left(\mathbf{x}_{k}\right)=\frac{1}{c} m\left(\mathrm{E}_{i, k}\right) \Delta\left(\mathrm{h}_{\mathrm{a}, k}\right), \\
\text { with } m\left(\mathrm{E}_{i, k}\right)=\frac{1.0121}{\sin \mathrm{E}_{i, k}+0.0121}, \quad \Delta\left(\mathrm{h}_{\mathrm{a}, k}\right)=2.4405 e^{-0.133 \times 10^{-3} \mathrm{~h}_{\mathrm{a}, k}} .
\end{gathered}
$$

The Zenit delay $\Delta$ is a function of the receiver antenna altitude $\mathrm{h}_{\mathrm{a}, k}$, and the mapping function $m$ depends on the satellite apparent elevation $\mathrm{E}_{i, k} . c$ is the speed of light.

The sensitivity vector of this model to the error-state vector can be given as

$$
\mathbf{h}_{\tau_{\mathrm{T}, i, k}}=\frac{\partial h_{\tau_{\mathrm{T}}, i, k}\left(\mathbf{x}_{k}\right)}{\partial \boldsymbol{\delta} \mathbf{x}_{k}}=-\frac{1}{c} m\left(\mathrm{E}_{i, k}\right) \Delta\left(\mathrm{h}_{\mathrm{a}, k}\right)\left[\begin{array}{c}
0.133 \times 10^{-3} \\
m\left(\mathrm{E}_{i, k}\right)
\end{array}\right]^{\mathrm{T}}\left[\begin{array}{c}
\frac{\partial \mathrm{h}_{\mathrm{a}, k}}{\partial \boldsymbol{\delta} \mathbf{x}_{k}} \\
\frac{\partial \sin \mathrm{E}_{i, k}}{\partial \boldsymbol{\delta} \mathbf{x}_{k}}
\end{array}\right],
$$

with

$$
\mathrm{h}_{\mathrm{a}, k}=\left\|\mathbf{r}_{\mathrm{a}, k}^{E}\right\|-\mathrm{R}_{\oplus}, \quad \sin \mathrm{E}_{i, k}=\left(\mathbf{e}_{\mathrm{a}, k}^{E}\right)^{\top} \mathbf{e}_{\rho, i, k}^{E},
$$

where $\mathbf{R}_{\oplus}$ is the Earth radius, $\mathbf{r}_{\mathrm{a}, k}^{E}$ is the receiver antenna position, $\mathbf{e}_{\mathrm{a}, k}^{E}$ is the unit direction from the ECEF origin to the receiver antenna, and $\mathbf{e}_{\rho, i, k}^{E}$, as in (45), is the unit range vector. The non-null partial derivatives in (65) are then

$$
\begin{aligned}
\frac{\partial \sin \mathrm{E}_{i, k}}{\partial \boldsymbol{\delta} \mathbf{r}_{k}^{E}}=\frac{\hat{\mathbf{e}}_{\mathrm{a}, k}^{E}}{\left\|\hat{\mathbf{r}}_{\mathrm{a}, k}^{E}\right\|}\left[\hat{\mathbf{e}}_{\rho, i, k}^{E} \times\right]^{2}-\frac{\hat{\mathbf{e}}_{\rho, i, k}^{E}}{\hat{\rho}_{i, k}}\left[\hat{\mathbf{e}}_{\mathrm{a}, k}^{E} \times\right]^{2}, \quad \frac{\partial \mathrm{h}_{\mathrm{a}, k}}{\partial \boldsymbol{\delta} \mathbf{r}_{k}^{E}}=\left(\hat{\mathbf{e}}_{\mathrm{a}, k}^{E}\right)^{\mathrm{T}}, \\
\frac{\partial \sin \mathrm{E}_{i, k}}{\partial \boldsymbol{\delta} \boldsymbol{\theta}_{k}^{E}}=\frac{\partial \sin \mathrm{E}_{i, k}}{\partial \boldsymbol{\delta} \mathbf{r}_{k}^{E}} \hat{\mathbf{C}}_{B_{k}}^{E_{k}}\left[\mathbf{l}_{\mathrm{a}}^{B} \times\right], \quad \frac{\partial \mathrm{h}_{\mathrm{a}, k}}{\partial \boldsymbol{\delta} \boldsymbol{\theta}_{k}^{E}}=\frac{\partial \mathrm{h}_{\mathrm{a}, k}}{\partial \boldsymbol{\delta} \mathbf{r}_{k}^{E}} \hat{\mathbf{C}}_{B_{k}}^{E_{k}}\left[\mathbf{l}_{\mathrm{a}}^{B} \times\right] .
\end{aligned}
$$

Acknowledgements The authors would like to thank ESA, Guidance, Navigation \& Control Section at the European Space Research and Technology Centre for granting the funding, through the Network/Partnering Initiative contract 4000111837/14/NL/MH, which enabled this research. In addition, the authors would like to thank Oliver Montenbruck, Markus Markgraf and Benjamin Braun from the German Space Operations Center (DLR) for the support to this work.

\section{References}

[1] B. Braun, M. Markgraf, and O. Montenbruck. "Performance analysis of IMU-augmented GNSS tracking systems for space launch vehicles". In: CEAS Space Journal 8.2 (2016).

[2] E. Burke, E. Rutkowski, and E. Rutkowski. "Vehicle Based Independent Tracking System (VBITS): A Small, Modular, Avionics Suite for Responsive Launch Vehicle and Satellite Applications". In: 6th Responsive Space Conference. 2008.

[3] A. J. van Dierendonck, J. B. McGraw, and R. G. Brown. "Relationship Between Allan Variances and Kalman Filter Parameters". In: 16th Annual PTTI Meeting. 1984. 
[4] V. Dishel and E. Mezhiritskiy. "Principals Of Integrated Ins/GLONASS+GPS GNC Systems For Space Launchers. Results Of Realized Missions And Future Prospects". In: 8th International ESA Conference on Guidance, Navigation \& Control Systems. 2011.

[5] J. A. Farrell. Aided Navigation - GPS with High Rate Sensors. McGraw-Hill, 2008.

[6] M. Giannini, M. Melara, and C. Roux. "Alts Localization System Of Vega Launcher: Vv02 Post-Flight Analysis \& Gps Issues For Future Hybrid Navigation”. In: 9th International ESA Conference on Guidance, Navigation \& Control Systems. 2014.

[7] B. Gibbs. Advanced Kalman Filtering, Least-Squares and Modeling. Wiley \& Sons, 2011.

[8] F. van Graas and A. Soloviev. "Precise Velocity Estimation Using a Stand-Alone GPS Receiver". In: Journal of the Institute of Navigation (2004).

[9] P. D. Groves. Principles of GNSS, Inertial, and Multisensor Integrated Navigation Systems. Artech House, 2008.

[10] X. Guochang. GPS Theory, Algorithms and Applications. 2nd Ed. Springer, 2007.

[11] A. Hauschild, M. Markgraf, et al. "Results of the GNSS Receiver Experiment OCAM-G on Ariane-5 flight VA 219". In: 6th European Conference for Aeronautics and Space Sciences. 2015.

[12] B. Hofmann-Wellenhof, H. Lichtenegger, and E. Wasle. GNSS - Global Navigation Satellite Systems. Springer, 2008.

[13] M. Markgraf and O. Montenbruck. "Phoenix-HD - A Miniature GPS Tracking System for Scientific and Commercial Rocket Launches." In: 6th International Symposium on Launcher Technologies. 2005.

[14] R. A. McKern. "A Study of Transformation Algorithms for Use in a Digital Computer". MA thesis. University of Illinois, 1968.

[15] O. Montenbruck and M. Markgraf. "Global Positioning System Sensor with InstantaneousImpact-Point Prediction for Sounding Rockets". In: Journal of Spacecraft and Rockets (2004).

[16] NATO Standardization Agreement (STANAG). Doc. 4294, Edition 1. NATO, 1993.

[17] B. W. Parkinson and J. J. Spilker Jr. Global Positioning System: Theory and Applications. Vol. 1. AIAA, 1996

[18] B. Polle, B. Frapard, et al. "Robust INS/GPS Hybrid Navigator Demonstrator Design for Launch, Re-entry and Orbital Vehicles". In: 7th International ESA Conference on Guidance, Navigation and Control Systems. 2008.

[19] M. Schlotterer. "Navigation System for Reusable Launch Vehicle". In: 31 st Annual AAS Guidance and Control Conference. 2008.

[20] S. Slivinsky, C. Nesbit, et al. "Development and Demonstration of a Ballistic Missile Range Safety Technology System". In: Journal of the Institute of Navigation (2002).

[21] S. R. Steffes. "Computationally Distributed Real-Time Dual Rate Kalman Filter”. In: Journal of Guidance, Control and Dynamics (2014).

[22] S. R. Steffes. "Development and Analysis of SHEFEX-2 Hybrid Navigation System Experiment". PhD thesis. University of Bremen, 2013.

[23] S. R. Steffes. "Real-Time Navigation Algorithm for the SHEFEX2 Hybrid Navigation System Experiment". In: AIAA Guidance, Navigation, and Control Conference. 2012

[24] S. R. Steffes, S. Theil, and M. A. Samaan. "Post-Mission Analysis Of Flight Results From The SHEFEX2 Hybrid Navigation System". In: 9th International ESA Conference on Guidance, Navigation \& Control Systems. 2014.

[25] S. Theil, S. R. Steffes, et al. "Hybrid Navigation System for Spaceplanes, Launch and ReEntry Vehicles". In: 16th AIAA/DLR/DGLR International Space Planes and Hypersonic Systems and Technologies Conference. 2009.

[26] J. Wendel, C. Schaile, and G. F. Trommer. "Direct Kalman Filtering of GPS/INS for Aerospace Applications". In: International Symposium on Kinematic Systems in Geodesy, Geomatics and Navigation. 2001.

[27] J. Wendel and G. F. Trommer. "Tightly coupled GPS/INS integration for missile applications". In: Aerospace Science and Technology 8 (2004).

[28] A. Williams, M. Villa, and J. Puig-Suari. "Platform Independent Launch Vehicle Avionics". In: 28th Annual AIAA/USU Conference on Small Satellites. 2014.

[29] B. Willms. "Space Integrated GPS/INS (SIGI) Navigation System for Space Shuttle". In: 18th Digital Avionics Systems Conference. 1999.

[30] J. Zhou, S. Knedlik, and O. Loffeld. "INS/GPS for High-Dynamic UAV-Based Applications". In: International Journal of Navigation and Observation (2012). 